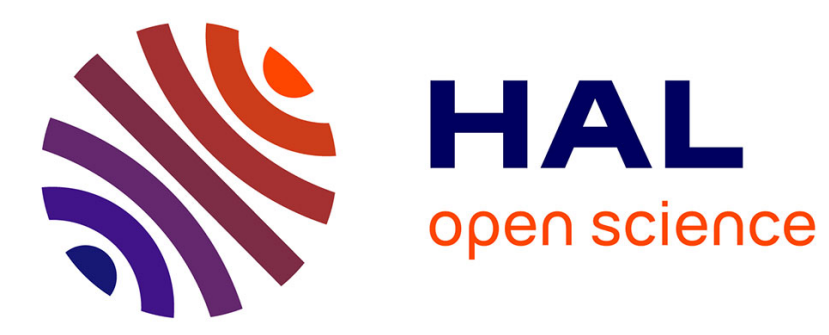

\title{
Le point sur les modulateurs spatiaux de lumière
}

G. Lebreton

\section{To cite this version:}

G. Lebreton. Le point sur les modulateurs spatiaux de lumière. Revue de Physique Appliquée, 1987, 22 (10), pp.1309-1320. 10.1051/rphysap:0198700220100130900 . jpa-00245682

\section{HAL Id: jpa-00245682 https://hal.science/jpa-00245682}

Submitted on 1 Jan 1987

HAL is a multi-disciplinary open access archive for the deposit and dissemination of scientific research documents, whether they are published or not. The documents may come from teaching and research institutions in France or abroad, or from public or private research centers.
L'archive ouverte pluridisciplinaire $\mathbf{H A L}$, est destinée au dépôt et à la diffusion de documents scientifiques de niveau recherche, publiés ou non, émanant des établissements d'enseignement et de recherche français ou étrangers, des laboratoires publics ou privés. 
Classification

Physics Abstracts

85.10PH - 85.60JPM

\title{
Le point sur les modulateurs spatiaux de lumière
}

\author{
G. Lebreton \\ GESSY, Université de Toulon et du Var, 639, boulevard des Armaris, 83100 Toulon, France
}

(Reçu le 13 novembre 1986, accepté le 6 mars 1987)

\begin{abstract}
Résumé. - Les nouvelles technologies qui apparaissent aujourd'hui pour la modulation spatiale de lumière font l'objet d'une revue critique. Les divers types de modulateurs sont classés du point de vue de leur utilisation dans des architectures de processeurs optiques: modulateurs unidimensionnels pour systèmes optiques tri-dimensionnels ou à ondes guidées; modulateurs bidimensionnels à adressage électronique ou optique ; mode d'opération analogique, binaire ou non linéaire. Une véritable révolution est observée en direction de «technologies compatibles ", où matériaux et techniques de fabrication tendent à devenir très similaires dans la réalisation de modulateurs de lumière ou de circuits électroniques à haut degré d'intégration. Il est suggéré que le fossé séparant les actuels processeurs hybrides opto-électroniques et les nouvelles portes logiques «tout-optiques" sera bientôt comblé par une nouvelle génération de convertisseurs parallèles électrons-photons totalement intégrés.
\end{abstract}

\begin{abstract}
A critical assessment is given on the now emerging technologies for the spatial modulation of light. The many types of actually developed light valves are reviewed, using a classification based on their utilization in optical architectures and systems : one-dimensional bulk or integrated devices ; two-dimensional modulators with electronical or optical addressing ; analog, binary or non-linear (including bistable) mode of operation. A coming revolution is stated towards "compatible technologies", where materials and manufacturing technologies tend to become very similar for the realization of light modulators and of highlyintegrated electronic circuits. It is suggested that the actual gap between the existing hybrid opto-electronic processors and the coming «all-optical» logic gates is going to be filled with a new class of fully-integrated electrons-photons parallel converters.
\end{abstract}

\section{Introduction.}

Trois principaux facteurs d'évolution concourent à un bouleversement observé ces dernières années dans la technologie des modulateurs spatiaux de lumière : le progrès des techniques, avec un début de production industrielle ; un marché élargi grâce à des applications diversifiées ; une nouvelle conception des systèmes de calcul optique.

Les progrès techniques sont issus de la recherche de nouveaux matériaux et surtout de la mise en œuvre récente des technologies de la micro-électronique : la mise en production industrielle de nombreuses barrettes ou matrices de photodétecteurs, le développement rapide de la production des lasers à semi-conducteurs, ont amorcé un mouvement qui, pour les modulateurs spatiaux, se résume essentiellement au passage du monocristal aux mosaïques à semi-conducteurs (qu'il s'agisse d'adressage électrique ou optique).
Le marché des modulateurs spatiaux était limité jusqu'à présent à la déflexion-modulation de faisceaux laser et à la réalisation de quelques processeurs spécialisés pour le traitement optique du signal (analyse spectrale ou convolution bidimensionnelle). Il s'ouvre aujourd'hui dans au moins quatre directions nouvelles: la connectique tridimensionnelle fixe ou programmable entre VLSI pour des architectures massivement parallèles, avec des possibilités de commutation («crossbar switch») utilisables aussi en télécommunications à fibres ou pour l'interconnexion de gros réseaux informatiques; le calcul matriciel numérique itératif à « engagement » (architectures semi-systoliques, semi-parallèles); les mémoires à accès parallèle (type CAM) associatives (intelligence artificielle) éventuellement «attentives » (automates cellulaires à connexion du type «nearest neighbour »); les architectures massivement parallèles pour le traitement d'images.

Au plan de la conception des systèmes optiques de 
calcul, les deux innovations fondamentales sont le passage au codage numérique parallèle et aux logiques à seuil d'une part (objet d'une étroite coopération avec les informaticiens pour la recherche algorithmique), et d'autre part l'utilisation simultanée de l'intégration sur les variables d'espace et de temps qui permet aujourd'hui l'utilisation efficace de modulateurs unidimensionnels (à ondes volumiques ou de surface) dans des architectures bi- ou tridimensionnelles.

La conjonction de ces trois facteurs d'évolution a depuis environ quatre ans fait naître une nouvelle génération de modulateurs spatiaux basés sur la micro-électronique, sans que soient totalement périmées les approches anciennes. Quelques années seront nécessaires pour que s'opère le tri des technologies, avec la mise en production industrielle des dispositifs présentant le meilleur rapport coût-performances. On tentera en conclusion de dégager les grands axes de cette évolution, mais la présentation qui suit se limite déjà (sauf mentions historiques jugées utiles à la compréhension) aux dispositifs faisant actuellement l'objet d'études importantes avec un début ou au moins un sérieux espoir de mise en production industrielle. Ont été omis pour cette raison certains des modulateurs recensés dans l'excellente revue d'A. Fisher [1, 2] à laquelle nous renvoyons pour une approche plus experte en physique des matériaux.

Il serait possible de passer en revue les modulateurs spatiaux de lumière en s'attachant au choix des matériaux utilisés ou du principe physique mis en œuvre dans les interactions photons-électrons. Le plan retenu ici correspond plutôt aux préoccupations du concepteur de systèmes optiques mettant en œuvre ces modulateurs : unidimensionnels ou bidimensionnels à adressage respectivement électronique ou optique. Pour ces derniers, on s'attachera en particulier aux modes de fonctionnement non linéaires (à seuil ou bistable), qui sont un objectif essentiel de la recherche actuelle pour un traitement optique en logique parallèle.

\section{Modulateurs unidimensionnels.}

Cette appellation traditionnelle mais inadéquate recouvre l'ensemble des modulateurs vectoriels spatio-temporels. Dans un conducteur électrique, la tension ou le courant constitue une dimension modulable en un point au cours du temps avec un seul axe de propagation spatiale ; on peut certes y multiplexer plusieurs fréquences temporelles, mais il ne s'agit que d'un codage créant une partition en sous-espaces. Dans une fibre optique, s'ajoute la possibilité de multiplexer $n$ longueurs d'ondes, donnant $n$ dimensions de modulation dans un espace géométrique à une dimension. Un modulateur transverse en optique intégrée peut, lui, être considéré comme ayant deux dimensions géométriques : l'axe de propagation optique et celui du modulateur. Dans les modulateurs acousto-optiques à onde volumique, il faut ajouter à l'axe géométrique de propagation la dimension transverse de la pupille, qui permet un multiplexage angulaire créant $m$ dimensions supplémentaires. Dans tous les cas cités, la modulation peut être soit en intensité, soit en amplitude complexe en éclairage cohérent ; la polarisation de la lumière, enfin, constitue une autre dimension de modulation.

1.1 Modulateurs volumiQues acousto-OPTIQUES. - Les remarques précédentes ont connu un début d'application avec l'emploi de ces modulateurs dans des architectures tridimensionnelles, grâce à l'utilisation simultanée de l'intégration sur les variables d'espace et de temps. Dans une revue très complète de ces nouvelles architectures largement développées au CALTECH, D. Psaltis [3] souligne combien il est crucial d'utiliser la troisième dimension géométrique effectivement (par la connectique tridimensionnelle), car c'est elle en général qui donne à l'optique un avantage clair sur les autres alternatives technologiques. Il ajoute que l'emploi de modulateurs 1-D en concurrence avec les modulateurs 2-D se justifie non seulement à cause de leur développement industriel très avancé, mais aussi parce que leur bande-passante est intrinsèquement plus élevée, ce qui compense la perte de parallélisme par rapport aux modulateurs 2-D. Un autre atout dans l'emploi des modulateurs 1-D est la généralisation récente des algorithmes systoliques ou «à engagement », permettant l'introduction de matrices (ou d'images) pour une multiplication bidimensionnelle, grâce au «produit extérieur » matriciel et aux algorithmes récents de produit binaire par convolution [4].

Commercialisés par de nombreux industriels, les modulateurs acousto-optiques à onde volumique ont pratiquement atteint la limite théorique de leurs performances, avec une bande passante de 0,1 à $\mathrm{GHz}$ (selon le choix des matériaux), une dynamique de 40 à $50 \mathrm{~dB}$ et un nombre de points résolus (produit durée-bande) de 1000 à 4000 . Deux innovations sont cependant à souligner chez Harris Corp. [5], qui commercialise depuis peu un modulateur temporel de 64 points en parallèles avec une bande de $200 \mathrm{MHz}$ par point (soit un débit de $12,8 \mathrm{GHz}$ ), ainsi qu'un déflecteur permettant la modulation spatiale parallèle de 64 canaux avec une résolution de 200 points par canal (bande passante $200 \mathrm{MHz}$ ). Ce dernier dispositif permet par exemple un codage spatial binaire bipolaire sur 32 digits qui peut se substituer aux processeurs analogiques utilisant précédemment un modulateur 1-D classique.

Une autre innovation importante pour les systèmes est l'utilisation, en amont des photodétecteurs, d'une pré-intégration temporelle sur cristal BSO, 
qui permet d'éliminer la composante continue préjudiciable aux convoluteurs à intégration temporelle [6]. Ce dispositif est applicable à tous les modulateurs spatiaux à déformation de surface (membrane, thermoplastiques), qui permettent de soustraire un niveau ajustable de la composante continue d'éclairement, durant une intégration temporelle, pour optimiser la dynamique de modulation.

1.2 ModulateUrs EN OPTIQUE INTÉGRÉE. - Les modulateurs électro ou acousto-optiques à guide d'onde plan n'ont pas atteint le niveau de performances de leurs homologues purement électroniques, en raison de la résolution limitée des lentilles géodésiques. Pour cette raison, ils ont été supplantés par les dispositifs volumiques pour le traitement du signal, sauf dans les cas privilégiant une miniaturisation extrême. Par contre, ils connaissent un nouvel essor comme interfaces de modulation ou commutation pour les dispositifs à fibre optique. Si leur emploi comme pré-processeurs numériques «à engagement » conserve de nombreux partisans [7], il semble qu'un domaine plus prometteur s'ouvre avec leur emploi comme modulateurs vectoriels dans des processeurs optiques tridimensionnels, comme en témoignent les trois exemples suivants.

Pour ses futures imprimantes à laser, Xerox [8] a développé le TIR (Total Internal Reflection), un modulateur électro-optique intégré de 5000 pixels adressés individuellement (sandwich VLSI - monocristal de niobate de lithium), dont la vitesse n'est limitée que par l'électronique. Le prototype actuel a une bande passante de $32 \mathrm{MHz}$ répartie en 16 canaux d'adressage, avec une tension de pilotage inférieure à $5 \mathrm{~V}$. Il s'agit d'une modulation de phase, et donc d'amplitude complexe en éclairage cohérent. Un autre dispositif prometteur [9], développé au MIT Lincoln Laboratory, utilise l'électro-absorption (effet Franz-Keldysh) dans une structure AsGa à transfert de charges. Une version à incidence optique normale s'est avérée limitée à $20 \mathrm{~dB}$ en dynamique, tandis que $40 \mathrm{~dB}$ ont été obtenus avec la version guidée avec un rendement de diffraction proche de $100 \%$ grâce à la plus grande longueur d'interaction, et une fréquence de modulation atteignant $1 \mathrm{GHz}$. Il est intéressant de souligner que pour ce dernier modulateur l'éclairement optimal correspond au proche infrarouge des diodes lasers (905 nm).

Il n'a pas été possible d'obtenir des informations sur le développement d'un modulateur étudié initialement par l'U.S. Navy, utilisant des ondes magnétostatiques de surface [10] sur matériau YIG (Yttrium Iron Garnet) pour moduler une rotation de polarisation lumineuse par effet Faraday entre 1 et $20 \mathrm{GHz}$. Les points faibles de ce dispositif sont le faible rendement de diffraction (limite théorique $4 \%$ ) et la difficulté d'obtenir de larges bandes passantes.
On peut rattacher aux modulateurs à ondes guidées deux domaines voisins qui font actuellement l'objet d'importantes recherches : barrettes de diodes émettrices, et projet PRIMO de Hughes Aircraft. Sous la pression d'une demande importante, on commence à voir apparaître des barrettes de diodes modulables : D. Psaltis [3] signale un prototype de 16 diodes laser, ainsi que la commercialisation d'une barrette de 128 diodes électroluminescentes modulables (de $20 \mu \mathrm{W}$ seulement) par Spectronix. De nombreux laboratoires utilisent provisoirement une ligne de fibres optiques connectées à des diodes laser, pour une entrée vectorielle à haut débit (potentiellement plusieurs dizaines de $\mathbf{G H z}$ ) et forte puissance. Il est également question de la sortie prochaine d'une matrice de diodes-laser en préparation au Japon.

Toute autre est l'approche de Hughes [11], avec son projet de processeur optique intégré PRIMO (Programmable Real-time Incoherent Matrix multiplier for Optical processing), qui utiliserait des plans d'adressage électro-optique à modulations unidimensionnelles orthogonales (cristal niobate de lithium) suivis d'une matrice de photodétecteurs pour intégration temporelle (Fig. 1). Des variantes de ce dispositif pourraient être spécialement construites pour des applications spécialisées, telle l'inversion de matrice utilisant l'algorithme de Faddeev.

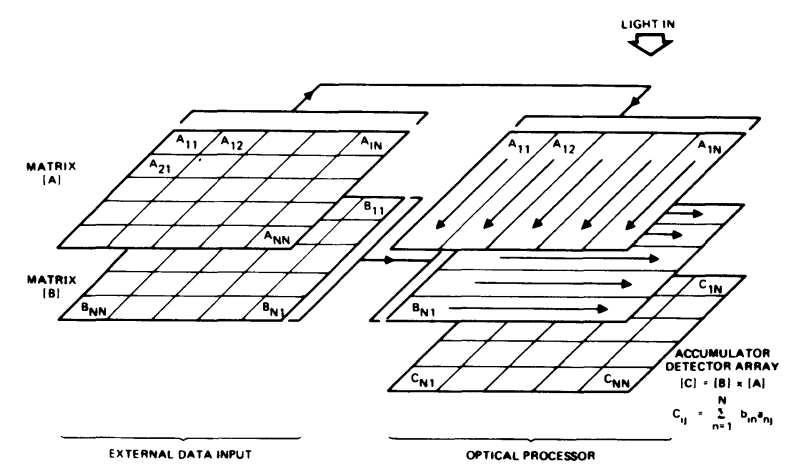

Fig. 1-Dispositif PRIMO : produit extérieur matriciel (reproduit d'après Réf. [11]).

[PRIMO architecture : matrix multiplication using outer product (from Ref. [11]).]

\section{Modulateurs bidimensionnels à adressage électro- nique.}

Les années 70 ont vu le développement de nombreux dispositifs inspirés des tubes cathodiques classiques, mais destinés à la projection sur grand écran d'images de télévision grâce au remplacement de l'écran phosphorescent par un modulateur lisible en réflexion par une puissante source lumineuse. Cette source pouvant toujours être un laser, on a accessoirement envisagé l'emploi de ces tubes comme 
« imageurs cohérents », permettant d'utiliser la transformation de Fourier bidimensionnelle pour l'analyse spectrale ou la convolution. Mais aucun de ces dispositifs n'avait la qualité de surface d'onde nécessaire et, lorsqu'on a su compenser ce défaut par conjugaison de phase, les progrès de l'informatique étaient suffisants pour effectuer ce type d'opération avec la bande passante des signaux Vidéo. De même que le tube cathodique est concurrencé par divers types d'écrans plats pour la visualisation de données ou graphiques, ces « imageurs cohérents» se voient aujourd'hui concurrencés par deux nouvelles familles de modulateurs spatiaux plans, utilisant un adressage matriciel par électrodes ou par transfert de charges. On peut aussi considérer comme imageurs l'association de modulateurs à adressage optique et de modulateurs-déflecteurs de faisceau laser.

\subsection{ADRESSAGE PAR FAISCEAU D'ÉLEC-} TRONS. - Les nouvelles architectures développées pour le calcul parallèle font beaucoup plus souvent appel à la directivité du faisceau laser qu'à sa cohérence. Les imageurs Vidéo actuellement commercialisés ont survécu grâce à une résolution améliorée et pour des applications demandant une grande luminosité. Essentiellement, il s'agit de l'imageur à film d'huile déformable développé par General Electric [12], dont une version est également produite par la société suisse Gretag [13], et de l'imageur Titus à effet Pockels conçu au LEP [14], dont la commercialisation a été reprise par la Sodern pour des applications militaires (simulateurs de vol ou affichage dans des salles de commandement opérationnel). Les améliorations en cours devraient permettre au Titus de viser le nouveau créneau des imageurs destinés aux processeurs optiques, dont l'objectif fixé aux USA par les utilisateurs militaires potentiels est de $1000 \times 1000$ points résolus avec une cadence image de $1 \mathrm{kHz}$ et une dynamique linéaire de 100 niveaux de gris.

Le tube cathodique laser développé en URSS [15, 16] ne peut ambitionner une cadence supérieure à 10 images/seconde, mais il a pour lui la résolution $(1000 \times 1000$ points selon une communication privée de A. Nasibov en 1982), la puissance $\left(5 \mathrm{~W} / \mathrm{cm}^{2}\right)$ et la diversité des longueurs d'ondes émises selon le matériau utilisé comme écran : $450 \mathrm{~nm}(\mathrm{ZnSe}), 500$ à $650 \mathrm{~nm}(\mathrm{CdSxSe} 1-\mathrm{x})$, $800 \mathrm{~nm}$ (GaAs). Rappelons qu'il s'agit d'une conversion du faisceau d'électrons en faisceau laser, avec un rendement de l'ordre de $15 \%$, une puissance lumineuse de $10 \mu \mathrm{W}$ pendant $100 \mathrm{~ns}$ pour un point de diamètre $10 \mu$ en émission multimode (largeur de raie $5 \mathrm{~nm}$ ) avec une divergence du faisceau d'environ $10^{\circ}$.

Deux dispositifs à adressage optique utilisant comme entrée un intensificateur de lumière (photocathode et galette de microcanaux), que nous ver- rons plus loin, font l'objet d'une variante remplaçant la photocathode par un faisceau d'électrons: le modulateur à effet Pockels développé par C. Warde au MIT [17] et celui à membrane déformable expérimenté au Naval Research Laboratory [18]. Vitesse et résolution insuffisante semblent condamner à brève échéance ce type de dispositifs, qui n'ont pas comme leurs homologues adressés optiquement l'avantage de permettre un fonctionnement non linéaire pour effectuer des opérations logiques.

\subsection{AdRESSAGE PAR ÉLECTRODES CROI-} SÉES. - Trois imageurs inégalement commercialisés représentent cette toute nouvelle génération. Il s'agit vraisemblablement d'une étape de transition car l'adressage, bien que permettant un accès aléatoire, ne peut s'effectuer en parallèle que sur un nombre de points égal à celui d'électrodes sur une dimension.

Le modulateur magnéto-optique réalisé par Litton [19] et récemment commercialisé par Semetex a connu un tel succès que le premier dispositif $(48 \times 48$ points), a été immédiatement suivi d'une version 128 $\times 128$ pixels au prix très compétitif actuellement de $16000 \$$, en attendant la sortie prochaine du modèle sans doute définitif de $512 \times 512$ éléments. Le modulateur est réalisé par croissance, sur un substrat a-magnétique, d'un film aimantable (bismuth substituted irongarnet) structuré en îlots (Fig. 2) dont

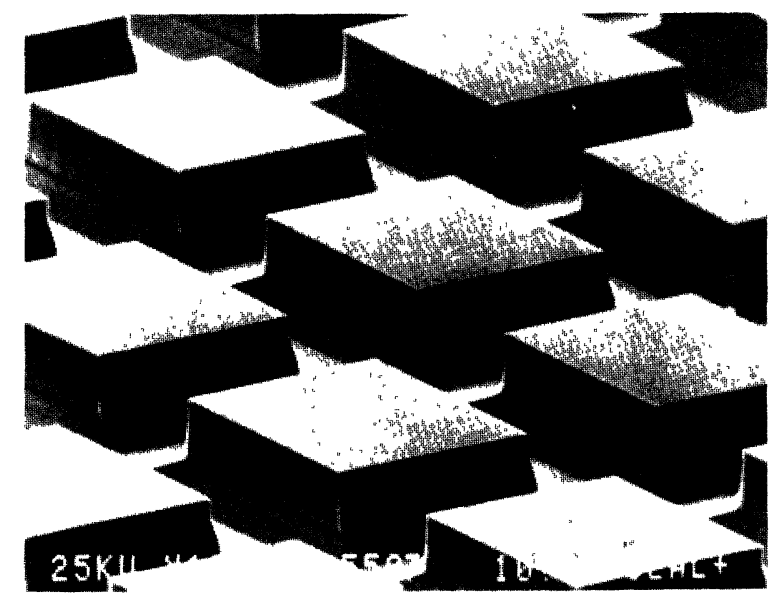

Fig. 2. - Imageur LITTON : photographie de la structure au microscope électronique (reproduit d'après Réf. [19]).

[LITTON device : scanning electron microscope picture of structured film (from Ref. [19]).]

chacun comporte un angle conducteur pour amorcer la croissance d'un domaine magnétique (Fig. 3). Cette croissance, non linéaire, fonctionne en mode tri-stable : état non magnétique, ou domaines dont la création nécessite un courant supérieur au seuil d'amorçage et dont la direction change de $180^{\circ}$ avec la polarité du courant. Le temps de commutation 


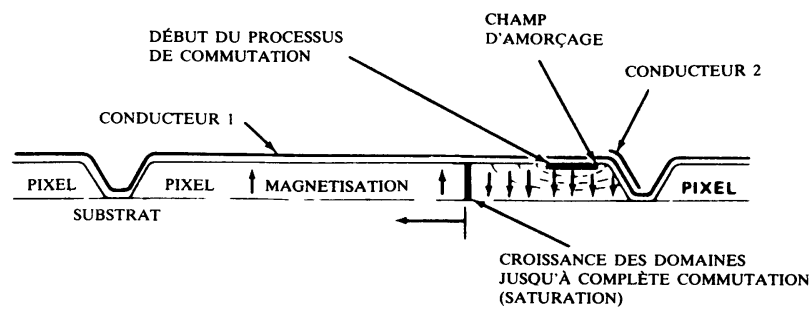

Fig. 3. - Imageur LITTON : processus de commutation d'un élément (reproduit d'après Réf. [19]).

[LITTON device: pixel switching process (from Ref. [19]).]

d'un pixel est inférieur à une microseconde. La modulation optique est une rotation de polarisation par effet Faraday, dont le maximum est actuellement de $32^{\circ}$ à $546 \mathrm{~nm}$ et $14^{\circ}$ seulement à $633 \mathrm{~nm}$. Le contraste est de $1000 / 1$ en éclairement monochromatique (20/1 en lumière blanche) et l'uniformité excellente $(1 \%)$, mais le rendement de diffraction optique ne dépasse pas $6 \%$. Ce point faible est dû au conflit entre rotation et absorption optiques, qui croissent simultanément avec l'épaisseur du film. La consommation électrique est inférieure à $1 \mu \mathrm{W}$ par pixel (carré de $76 \times 76 \mu$ ). Une version à l'étude pourrait permettre un codage sur plusieurs digits en utilisant un sandwich de plusieurs modulateurs d'épaisseur réduite.

Un modulateur également prometteur, réalisé par Texas Instruments [20], utilise un miroir déformable (membrane métallisée). Les quelques prototypes de la première version, réservés à l'évaluation des performances, utilisent un adressage par électrodes et comportent $128 \times 128$ pixels carrés au pas de $51 \mu$ (Fig. 4) avec un transistor MOS par élément
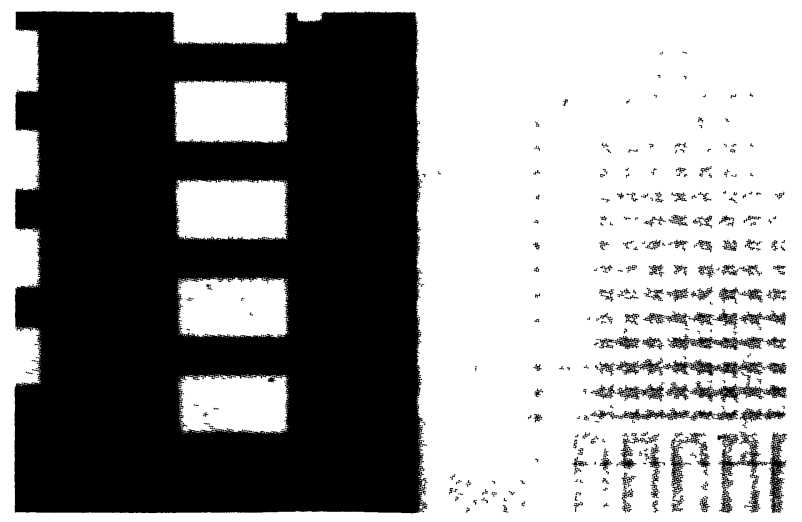

Fig. 4. - Micro-photographie d'un angle du dispositif à miroir déformable (DMD) de TEXAS INSTRUMENTS (reproduit d'après Réf. [20]).

[Photomicrograph of the TEXAS INSTRUMENTS Deformable Mirror Device (DMD) : corner view (from Ref. [20]).]

REVUE DE PHYSIQUe APPLIQUÉE. - T. 22, N` 10, OCTOBRE 1987
(Fig. 5). Bien que le temps de réponse de la membrane soit de $25 \mu \mathrm{s}$, la vitesse d'adressage des lignes n'est limitée que par le délai de propagation (environ $500 \mathrm{~ns}$ par ligne). La modulation de phase en éclairement cohérent donne une efficacité de diffraction de $8 \%$ et un rapport signal/bruit de $57 \mathrm{~dB}$. Un mode d'opération binaire est également possible en inclinant le modulateur par rapport à l'axe optique ; le rendement lumineux est alors d'environ $40 \%$ (taux de surface réfléchissante).

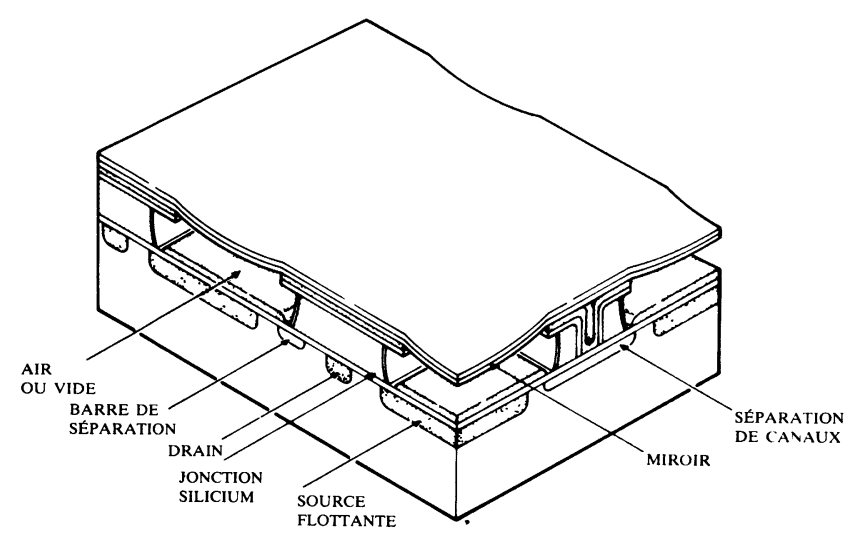

Fig. 5. - DMD de TEXAS INSTRUMENTS : structure des cellules élémentaires (reproduit d'après Réf. [20]).

[TEXAS INSTRUMENTS DMD : perspective view of elementary cells (from Ref. [20]).]

Un modulateur à performances limitées mais au très faible coût est celui qui équipe la télévision de poche japonaise largement commercialisée aux USA par Citizen et Radio-Shack pour un prix maximum de 130 \$. Il s'agit d'une cellule nématique twistée, avec une grille d'électrodes adressant $148 \times$ 122 pixels au pas de $0,22 \times 0,37 \mathrm{~mm}$, qui a été utilisée avec succès en traitement optique cohérent (avec un contraste de 20/1 après de légères modifications) par de nombreux laboratoires comme le MICOM [21] (U.S. Army Missile Command) et l'ERIM [22] (Environmental Research Institute of Michigan).

\subsection{ADRESSAGE PAR REGISTRES A TRANSFERT DE} CHARGES (CCD). - La version définitive du modulateur à miroir déformable développé par Texas Instruments cité au paragraphe précédent, qui comportera $1024 \times 1024$ pixels, utilisera une ligne d'entrée $\mathrm{AsGa}$ à $1,3 \mathrm{GHz}$, connectée à des colonnes de CCD classique au silicium, avec un dispositif original permettant le transfert parallèle instantané de l'image ainsi chargée sur le miroir électro-optique qui conservait en mémoire l'image précédente. Selon une communication privée, l'étude complète du dispositif est achevée et on attend actuellement la décision de sa mise en production ; mais il s'agit au 
départ d'un dispositif coûteux (nécessitant un circuit à 28 couches).

Hughes Aircraft prépare la mise en production (pas avant deux ans) d'un imageur à cristal liquide adressé par CCD, utilisant un transistor MOS par pixel, avec une grille de microdiodes pour bloquer la diffusion des charges [23]. Comme pour le modulateur de Texas Instruments, le transfert des charges sur le cristal liquide est effectué en parallèle : on peut ainsi charger une nouvelle image pendant la durée nécessaire à l'effacement de la précédente (relaxation du cristal liquide). Des prototypes de 256 $\times 256$ pixels fonctionnent actuellement avec une bande passante atteignant $20 \mathrm{MHz}$ et un contraste de 20 à 50/1. Le cristal liquide utilise l'effet de champ hybride (rotation de $45^{\circ}$ ) pour une lecture optique en réflexion.

Une toute nouvelle structure particulièrement prometteuse est actuellement étudiée au MIT Lincoln Laboratory [24]. Il s'agit d'un sandwich utilisant deux super-réseaux à puits quantiques GaAs/ AlGaAs, l'un pour la modulation (effet d'électroabsorption optique récemment découvert au laboratoire Bell AT \& T), l'autre pour la matrice d'adressage CCD. Ce dernier dispositif, mis au point au MIT, se distingue du MOS-CCD classique par une plus grande mobilité des charges : vitesse et efficacité de transfert accrues, grâce au confinement des charges dans une couche AsGa non dopée séparée de la zone de création des charges. Un premier prototype expérimental de 16 pixels, réalisé en 1985 , fonctionne avec une horloge à $1 \mathrm{MHz}$ et une tension maximale de $10 \mathrm{~V}$ pour la longueur d'onde optique optimale choisie $(870 \mathrm{~nm})$.

\subsection{AdRESSAGE PAR DÉFLEXION/MODULATION DE} FAISCEAU LASER. - Les dispositifs d'adressage par scanning laser, utilisant un modulateur temporel et des déflecteurs $(x, y)$, sont utilisés aujourd'hui pour de nombreuses applications industrielles des lasers. Toutes les combinaisons sont possibles entre modulateurs électro- ou surtout acousto-optiques et miroirs vibrant en résonance ou miroirs tournants à facettes, selon les performances recherchées. Les distorsions géométriques sont aisément compensées par des dispositifs d'asservissement. Une variante récemment perfectionnée par Xerox [25] est le dispositif Scophony (qui doit son nom à un ancêtre des années 30). Sa particularité est d'associer un déflecteur acousto-optique avec un miroir à facettes tournant en sens inverse, en synchronisant la durée d'emploi d'une facette avec le temps d'accès du déflecteur acousto-optique. Ce dernier est utilisé alors en modulateur spatial, donnant en projection une image fixe ; chaque facette du miroir écrit un segment de la ligne. Le miroir étant placé dans le plan de Fourier du modulateur, le signal adressé peut être filtré en BLU ; en outre, les impulsions successives de signaux numérisés sont codées en opposition de phase pour réduire leur interaction : on gagne ainsi un facteur 4 en résolution.

Depuis de nombreuses années, on réalise de grands panneaux de visualisation à cristal liquide smectique, selon un processus mis au point par Bell Laboratories [26] et exploité depuis par Thomson CSF en particulier [27]. Il s'agit d'une transition smectique-nématique à mémoire, commandée par échauffement local avec un laser infrarouge et assistée par un champ électrique. Une version très performante est aujourd'hui commercialisée par Laser-Scan Labs en Grande-Bretagne, sous le nom de HRD-1 : elle utilise un simple laser $\mathrm{He}-\mathrm{Ne}$ de $7 \mathrm{~mW}$, avec une résolution de $5 \mu$ et un maximum de $32000 \times 32000$ points. Des variantes de ce dispositif seraient intéressantes pour les applications compactes ne nécessitant pas le renouvellement rapide de toute l'image.

Pour l'adressage rapide, un autre procédé, expérimenté chez Itek dès 1978 pour le PROM [28], ne semble guère utilisé actuellement bien que les progrès techniques récents en permettent un emploi aisé. Il consiste à introduire une ligne du signal dans une cuve acousto-optique et à la projeter par un laser pulsé («snapshot») pour figer la translation. La version d'Itek, avec un modulateur acoustooptique de bande $50 \mathrm{MHz}$ et temps d'accès $10 \mu \mathrm{s}$, utilisait des impulsions de 20 ns. Aujourd'hui, avec des composants courants, on pourrait ainsi atteindre un débit d'entrée de $1 \mathrm{GHz}$ pour l'écriture des lignes (avec une déflexion orthogonale lente entre chaque ligne). Un tel dispositif est particulièrement intéressant pour le traitement cohérent d'antennes, car il est le seul procédé permettant de contrôler avec une grande précision la phase du signal spatial écrit sur l'imageur.

Cette technique de «snapshot» pourrait s'appliquer en particulier à l'adressage d'un film thermoplastique du type utilisé pour les caméras holographiques, selon une technique expérimentée en coopération par le GESSY et l'Université de Munich [29]. Il serait possible de réaliser un défilement continu du film avec inscription et lecture, avec une résolution pouvant atteindre $750 \mathrm{lp} / \mathrm{mm}$. Le procédé consiste à décharger localement par l'éclairement un réseau de charges à $750 \mathrm{lp} / \mathrm{mm}$ déposé au préalable. Actuellement, il n'existe aucun projet pour la réalisation industrielle d'un tel imageur à ultra-haute résolution. L'emploi d'un film mobile est rendu délicat par la fragilité mécanique de la couche thermoplastique ; quant à celui d'une plaque fixe, il se heurte à la fatigabilité du matériau : après une tentative peu fructueuse du ERIM dans les années 70 [30], des recherches en cours au C.N.E.T. pour accroître le nombre de cycles d'utilisation ont fait l'objet d'une communication à la « Journée d'Etude». 


\section{Modulateurs bidimensionnels à adressage optique.}

Nous ne reviendrons pas sur les caméras holographiques actuellement commercialisées, utilisant une plaque thermoplastique (NRC) ou un film (Rottenkolber, Optilas). Elles constituent cependant des détecteurs quadratiques linéaires avec seuil, parfaitement utilisables pour le traitement optique. L'ensemble des autres modulateurs 2-D à adressage optique peut être classé en trois catégories : les monocristaux, développés surtout dans les années 70 en recherchant une modulation linéaire de dynamique aussi grande que possible ; les dispositifs utilisant une matrice discrète d'amplificateurs électroniques, qui constituent la nouvelle génération actuellement développée pour le traitement non linéaire (logique à seuil) ; enfin, les dispositifs utilisant la bistabilité pour réaliser des matrices d'opérateurs logiques.

\subsection{MONOCRISTAUX ET MATÉRIAUX HOMOGÈ-} NES. - Leur étude était d'abord destinée à la réalisation de convertisseurs incohérents-cohérents à grande dynamique et haute résolution, pour les traitements optiques basés sur la transformation de Fourier. Leur développement en ce sens est resté difficile, en raison de l'étroitesse du marché des applications compétitives.

On peut citer pour mémoire les meilleurs dispositifs actuellement abandonnés : Phototitus du LEP, associant un photoconducteur (Se amorphe) et un KDP refroidi [31] ; gamma-Ruticon (photoconducteur-miroir élastomère), dont la résolution atteignait pourtant $80 \mathrm{lp} / \mathrm{mm}$ à $50 \%$ de modulation, mais avec une fonction de transfert passe-bande non plate [32] ; photodichroïques (alcalo-halogènes type $\mathrm{KCl}$ ou $\mathrm{NaF}$ ), de très haute résolution, mais pour lesquels les problèmes de vieillissement, non-uniformité et faible sensibilité n'ont pas été surmontés [33].

$\mathrm{Ne}$ sont également plus développés sous leur forme initiale les nombreux sandwiches photoconducteur-PLZT [34], qui ont cependant permis de maîtriser les divers processus de modulation des céramiques ferroélectriques : FERPIC de Bell Labs (biréfringence) ; CERAMPIC (modulation de diffusion lumineuse) et FERICON (déformation de surface) chez Sandia; FEWSIC (transition de phase AFE-FE) expérimenté au Japon.

La commercialisation du PROM (Pockels ReadOut Modulator), utilisant un cristal BSO (photoconducteur et biréfringent électrique) entre électrodes parallèles, a été récemment abandonnée par Itek faute de clientèle (en raison des défauts d'uniformité [35]), mais se poursuit au Japon avec Sumitomo pour une utilisation binaire à seuil plutôt qu'analogique [36]. Le point faible du PROM est surtout le faible rendement de diffraction (inférieur à $0,1 \%$ ). Le PRIZ [37], variante développée en URSS avec une orientation différente des axes cristallins, a des performances comparables en résolution à $50 \%$ de modulation $(8 \mathrm{lp} / \mathrm{mm})$ et en sensibilité $(200 \mathrm{~mJ} /$ $\mathrm{cm}^{2}$ ), mais un rendement de diffraction moins mauvais (proche de $2 \%$ ).

En dépit de sa lenteur, le sandwich photoconducteur-cristal liquide de Hughes-Aircraft [38] connaît depuis près de 10 ans un véritable succès commercial, bien que la version à haute planéité pour l'optique cohérente reste d'un prix élevé. La sensibilité est suffisante pour un adressage par tube cathodique, la cadence image atteint $8 \mathrm{~Hz}$ à pleine modulation (dynamique $25 \mathrm{~dB}$, mais non-uniformité $20 \%$ ) et la résolution est de $16 \mathrm{lp} / \mathrm{mm}$ (à $50 \%$ de modulation). Le cristal liquide est sans mémoire (nématique twisté à $45^{\circ}$, utilisé en réflexion).

Des performances tout à fait comparables au modulateur de Hughes ont été obtenues avec une variante utilisant comme photoconducteur un monocristal BSO au lieu d'une couche de CdS. L'uniformité est bien meilleure, mais au prix d'une perte de sensibilité (d'un facteur 5 environ). Expérimenté en URSS [39] et à Thomson-CSF [40], ce dispositif serait en voie de commercialisation en GrandeBretagne.

Une famille de modulateurs extrêmement simples à réaliser et donnant lieu à un nombre croissant d'applications est celle des cristaux à conjugaison de phase, type BSO ou titanate de baryum. D'abord utilisés pour l'holographie en temps réel, dans une configuration proposée par J. P. Huignard [41], ces modulateurs sont de plus en plus utilisés pour le couplage d'ondes optiques, qui en fait de véritables amplificateurs opérationnels [42] dans une boucle optique, utilisables comme amplificateurs d'image [43] avec une onde auxiliaire jouant le rôle de « pompe ». En couplage à trois ondes, ces modulateurs permettent également de réaliser les quatre opérations logiques [44], grâce à une amplification non linéaire à seuil, avec des temps de réponse qui ne sont limités que par l'énergie d'inscription (limite physique de l'ordre de la picoseconde).

Une récente utilisation des $\mathrm{BSO}$ ou $\mathrm{BGO}$, dans la même configuration que pour l'holographie en temps réel, est le PICOC (Photo refractive Incoherent Image Converter), qui utilise l'inscription séparée d'une trame (modulateur passe-bande) et de l'image [45]. Ce processus est analogue à celui déjà évoqué pour les thermoplastiques [29], avec une rapidité très supérieure, une résolution presque comparable (10 à $400 \mathrm{lp} / \mathrm{mm}$ ), mais une mémoire très courte (quelques ms) et une sensibilité 10 fois moins bonne.

Un imageur à gel déformable est actuellement étudié en Suisse, à l'Université de Neuchâtel. Il utilise un peigne d'électrodes pour créer un réseau de phase lu en réflexion, localement effacé par l'éclairement incident [46]. Il s'agit d'un dispositif linéaire avec possibilité de modulation binaire 
(dépassement du seuil d'effacement), de contraste supérieur à 40 (uniformité $15 \%$ ). La résolution atteindrait $10 \mathrm{lp} / \mathrm{mm}$, avec une sensibilité de quelques $\mathrm{mJ} / \mathrm{cm}^{2}$ et une cadence image maximale de $25 \mathrm{~Hz}$.

A cette catégorie des modulateurs à adressage optique sans assistance électronique, il faut rattacher le cas très particulier du réseau à pas variable développé par Hughes Aircraft en utilisant des cristaux liquides nématiques $[47,48]$ avec une couche photoconductrice de ZnS. Basé sur les instabilités électro-hydrodynamiques, ce modulateur code l'intensité d'éclairement en chaque point en un réseau de phase local unidimensionnel parallèle à l'orientation planaire des molécules du cristal liquide. Le processus est lent (1 s), mais la bande de fréquence couvre une octave $(300-600 \mathrm{lp} / \mathrm{mm})$ en 32 niveaux avec un éclairement de 4,5 à $9 \mathrm{~mW} /$ $\mathrm{cm}^{2}$ et une résolution de $256 \times 256$ points dans une pupille de $50 \times 50 \mathrm{~mm}$. Les difficultés restant à surmonter sont la durée de vie (pilotage par tension continue, provoquant une ionisation progressive), qui demande un cristal très pur, et les phénomènes anciennement connus de «désinclinaisons » (sauts locaux de la phase spatiale). L'intérêt du dispositif est la réalisation aisée des fonctions logiques par filtrage spectral dans le plan de Fourier. D'autre part, une application importante est aujourd'hui l'emploi comme réseau de diffraction pour la connectique tridimensionnelle programmable entre VLSI.

\subsection{MATRICE D'AMPLIFICATION ÉLECTRONI-} QUE. - Cette appellation regroupe deux familles très différentes: l'une, déjà classique, utilise un intensificateur électronique d'images (photocathode et galette de microcanaux) ; l'autre, plus significative de la nouvelle génération, utilise un sandwich VLSI - modulateur optique.

Le convertisseur d'images à micro-canaux développé depuis 10 ans par C. Warde au MIT a aujourd'hui évolué [49] vers l'emploi en modulateur non linéaire à seuil (« hard clipping »), grâce au contrôle de l'émission secondaire par une grille, pour la réalisation de fonctions logiques. Sous le brevet de ce dispositif, la firme Hamamatsu a d'abord commercialisé une caméra [50] à faible résolution ( $3 \mathrm{lp} / \mathrm{mm}$ à $50 \%)$ mais dont la sensibilité atteint la limite quantique $\left(5 \mathrm{~nJ} / \mathrm{cm}^{2}\right)$, utilisant une photocathode $\mathrm{S} 20$ et un cristal de niobate de lithium (effet Pockels) avec une cadence image inférieure à $13 \mathrm{~Hz}$. Un modèle plus récent y adjoint trois fonctions originales pour le prétraitement d'images: zoom électronique, translation électro-statique $x-y$, et rotation de l'image autour de l'axe optique par application d'un champ magnétique [51].

Un troisième dispositif à tube intensificateur est actuellement expérimenté au NRL [52]. L'originalité est de remplacer le cristal électro-optique par une membrane déformable de nitrocellulose (d'épaisseur $40 \mathrm{~nm}$ ) déposée après diverses couches intermédiaires sur la galette de microcanaux et métallisée par une couche d'oxyde d'indium (Fig. 6). Le miroir ainsi métallisé prend directement l'empreinte de la galette de microcanaux, ce qui évite les problèmes de focalisation des précédents dispositifs dont la résolution était très inférieure. Les autres caractéristiques, y compris le fonctionnement non linéaire comme opérateur logique à seuil, sont celles du dispositif précédent de C. Warde. L'objectif du développement en cours est une cadence image de $1 \mathrm{kHz}$ avec une résolution de 1 million de pixels.

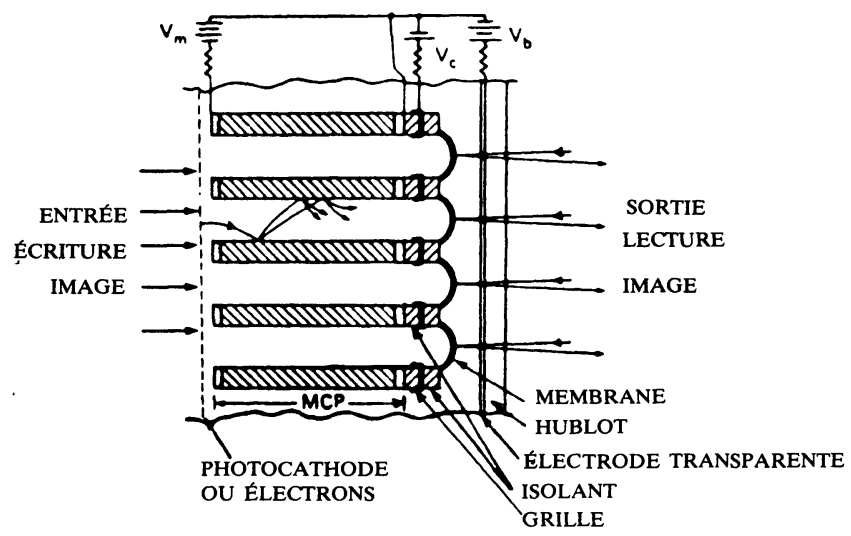

Fig. 6. - Modulateur de Lumière à Membrane PhotoExcitée, MLMPE (reproduit d'après Réf. [52]).

[Photo-Emitter Membrane Light Modulator, PEMLM (from Ref. [52]).]

La famille nouvelle des modulateurs adressés par photo-VLSI est représentée par quatre prototypes en voie de développement.

Chez Hughes, la matrice CCD-cristal liquide présentée au paragraphe 2.3 est aussi utilisée sans adressage $\mathrm{CCD}$, en transformant la matrice de transistors MOS au silicium qui lui sert de relais en photo-transistors [23].

Chez Texas Instruments [53], la même modification transforme le modulateur à membrane déformable en remplaçant l'adressage CCD [20] par un adressage optique. Le prototype actuel comporte $128 \times 128$ pixels au pas de $25 \mu$. Les temps d'écriture et d'effacement sont respectivement de 25 et $40 \mu \mathrm{s}$. La sensibilité, $2 \mathrm{~mJ} / \mathrm{cm}^{2}$ à pleine modulation, est 10 fois meilleure que celle du sandwich photoconducteur/cristal liquide de Hughes adressable par tube cathodique. Les longueurs d'onde utilisées sont de $520 \mathrm{~nm}$ pour l'écriture et $820 \mathrm{~nm}$ pour la lecture (diode laser). Un prototype de $256 \times 256$ pixels est en préparation pour la conversion linéaire incohérente-cohérente d'images à grande dynamique. Un fonctionnement linéaire à seuil serait sans doute possible en dépassant le seuil linéaire de fonctionne- 
ment, ce qui provoque une forte dépression brusque de la membrane sans fatigue apparente (communication privée).

Une technologie nouvelle actuellement étudiée chez TRW en coopération avec Transensory Devices [54] privilégie la facilité de construction, en remplaçant la membrane déformable par des pastilles carrées de $\mathrm{SiO}_{2}$ suspendues par un angle et larges de $80 \mu$ (Fig. 7), ayant un temps de réponse de $1 \mu$ s. La

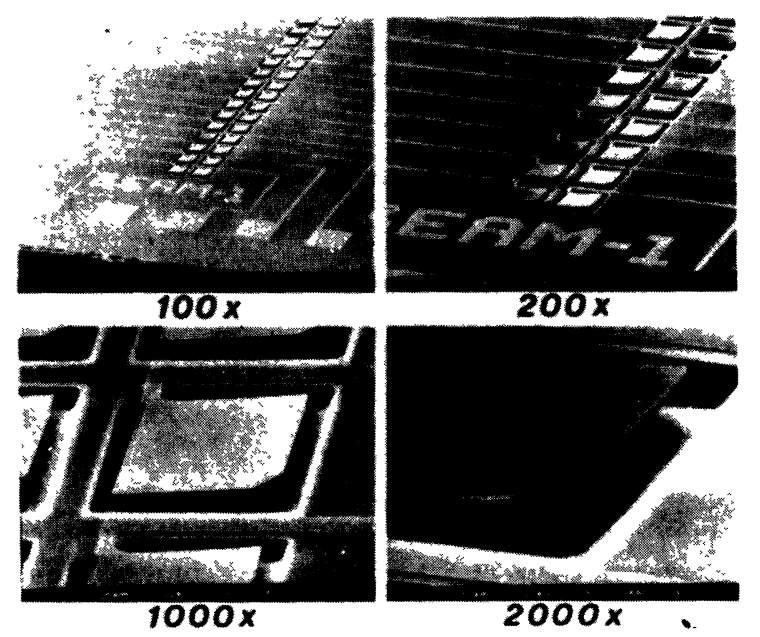

Fig. 7. - Photographie au microscope électronique du modulateur matriciel de TRW (reproduit d'après Réf. [54]).

[Scanning electron microscope picture of the TRW modulator array (from Ref. [54]).]

technologie utilisée étant totalement compatible avec celle des circuits intégrés électroniques (à substrat silicium), on peut associer ces pastillesmodulateurs avec des transistors de pilotage adressés optiquement ou électroniquement. Une utilisation envisagée est la lecture en parallèle de matrices de photodétecteurs (en fonctionnement linéaire), permettant par exemple la ré-injection du signal en boucle tout-optique. Un mode de fonctionnement binaire déjà expérimenté (en appliquant une tension supérieure au seuil de fonctionnement linéaire) permet l'utilisation de ce dispositif comme matrice d'opérateurs logiques à seuil.

Enfin, un effort considérable est en cours à l'UC de San Diego [55] pour la réalisation d'une matrice phototransistors-PLZT de $1000 \times 1000$ éléments (Fig. 8). Le phototransistor, d'une structure nouvelle et conçu spécialement, peut commuter une tension de $100 \mathrm{~V}$ (pour la pleine modulation) à $1 \mathrm{GHz}$. Les compromis nécessaires entre résolution et dissipation thermique conduisent à un développement séparé des dispositifs destinés à la modulation linéaire (dynamique de 1000 ) ou binaire (contraste limité à 10/1). Une structure en transmission (écriture et éclairement de lecture du même côté) est envisagée dans un premier temps; avec une cadence image

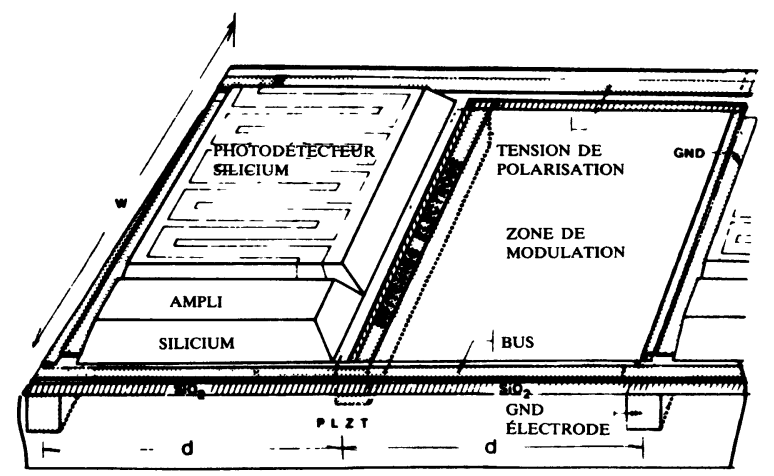

Fig. 8. - Modulateur matriciel Si/PLZT : schéma d'une cellule élémentaire (reproduit d'après Réf. [55]).

[Si/PLZT array : 3-D layout of a unit cell (from Ref. [55]).]

pouvant atteindre 1 à $2 \mathrm{kHz}$ en mode linéaire et 20 à $40 \mathrm{kHz}$ en mode binaire, et des sensibilités escomptées de 2000 et $120 \mathrm{~nJ} / \mathrm{cm}^{2}$ respectivement. Une structure en réflexion permettrait théoriquement des cadences beaucoup plus élevées mais jugées inutiles actuellement.

3.3 Modulations bistables. - Bien que de tels modulateurs aient été réalisés avec des cristaux liquides [56], leur intérêt en tant qu'opérateurs logiques parallèles est lié à la rapidité, qui nécessite l'emploi de matériaux non linéaires solides. Si la recherche active de matériaux organiques a déjà mis en évidence des non-linéarités dix fois plus fortes à réponse également rapide [57], les matériaux actuellement utilisés sont des semi-conducteurs. Trois laboratoires travaillent actuellement à la réalisation de modulateurs bidimensionnels bistables : à l'Université Heriot-Watt en Ecosse (avec le soutien d'un programme de la CEE et du SDI) ; au laboratoire central de Bell AT \& T (Holmdel, NJ) ; et au Optical Science Center de Tucson.

Le dispositif de Heriot-Watt utilise comme cellule de base un étalon de Fabry-Perot avec comme milieu non linéaire le $\mathrm{ZnSe}$, déjà expérimenté avec trois cellules déposées sur un même substrat mais connectées en cascade pour le fonctionnement en porte logique tout-optique [58]. L'objectif actuel est la réalisation d'une matrice de $100 \times 100$ cellules. La difficulté est la forte puissance optique nécessaire à l'onde optique de « pompe »; le faisceau modulateur proprement dit a besoin d'une puissance 40 fois plus faible environ pour déclencher la commutation. La modulation lumineuse en sortie a un contraste de $2 / 1$, suffisant pour permettre la commande d'autres portes en cascade. La miniaturisation des cellules devrait réduire d'un facteur 10 la consommation actuelle ( $2 \mathrm{~mW}$ par cellule). Le pas minimal imposé par la diffusion de charges pour l'indépendance des éléments modulateurs est de $0,3 \mathrm{~mm}$, ce qui devrait permettre une dissipation thermique suffisante pour des temps de commutation de $10 \mu \mathrm{s}$. Dans l'état 
actuel des performances, on prévoit qu'un laser de $3 \mathrm{~W}$ sera cependant nécessaire au fonctionnement d'un prototype de $100 \times 100$ éléments.

Le projet de modulateur de Bell AT \& T est basé sur une structure de super-réseau à puits quantique GaAs/AlGaAs, pour laquelle une énergie de commutation de $3 \mathrm{pJ}$ a été mesurée à $82 \mathrm{MHz}$ [59]. L'équipe de $H$. Gibbs (Centre récemment créé à l'Université de Tucson par la NSF pour le développement des composants optiques actifs) a récemment montré [60] que la résonance excitonique existait également à température ambiante dans le matériau AsGa et permettait une énergie de commutation aussi faible que la structure à puits quantiques de AT \& T. Par contre, celle-ci présente d'autres phénomènes physiques [61], permettant des applications aussi diverses que l'amplification optique («fourwave mixing » piloté par une diode laser de faible puissance), la réalisation de diode laser GaAs à mode bloqué, la modulation temporelle de lumière (temps de réponse inférieur à $100 \mathrm{ps}$ ), ou enfin une boucle électro-optique intégrée SEED (self electrooptic device) effectuant directement la conversion photon-électron ou réciproquement. C'est ce dernier effet SEED qui, sous une polarité électrique inverse, produit sans l'aide d'une cavité résonante le phénomène de bistabilité utilisé pour le modulateur d'AT \& T, pour lequel la difficulté reste l'intégration de nombreuses cellules sur un même substrat (complexité des couches à déposer). Actuellement, un prototype de $5 \times 5$ éléments seulement est en cours d'essais. Une cellule du même type, spécialement optimisée, a récemment été expérimentée à Tucson [62] en utilisant deux diodes-laser à $830 \mathrm{~nm}$ $(1 \mathrm{~mW}$ pour la commutation et $15 \mathrm{~mW}$ pour la pompe), ce qui confirme la crédibilité croissante de ce type d'opérateur logique.

C'est dans ce même Centre de Tucson qu'a été récemment fabriqué le premier prototype de matrice $100 \times 100$ à étalon GaAs/AlGaAs [63], avec des cellules de $9 \times 9 \mu$. Les premiers tests ont donné une énergie de commutation de $1 \mathrm{~nJ}$ avec des cycles de $0,5 \mathrm{~ns}$ par porte, mais l'uniformité n'est pas suffisante actuellement pour permettre une utilisation simultanée de toute la matrice.

Un autre type de structure non linéaire à semiconducteur est actuellement étudié par HewlettPackard Labs. [64], utilisant un seul matériau AsGa en couches alternées de dopage $n$ et $p$ séparées par des couches intrinsèques. Les premières expérimentations confirment les propriétés physiques escomptées, tout à fait similaires à celles du SEED d'AT \& T.

Le faible taux actuel de modulation en sortie des portes bistables rend pour l'instant difficile la mise en cascade d'un grand nombre de portes. Une solution possible serait l'effet de bistabilité externe (électro-optique) à fort gain récemment expérimenté par GTE Labs. [65] dans une structure diodelaser/amplificateur InGaAsP/InP. Avec une énergie de commutation très faible (quelques picoJoules) en une nanoseconde et un gain de 100 sur le signal optique de commutation, ce nouveau dispositif, possible également avec d'autres types de lasers semiconducteurs à émission transverse, ouvre une voie nouvelle qui permettrait d'éviter l'emploi de lasers à forte puissance pour la commande d'un nombre élevé de portes optiques bistables.

D'autres approches sont possibles, comme celle proposée par A. Lohmann [66] où la cavité bistable module non l'intensité de la lumière mais sa direction linéaire de polarisation entre deux états orthogonaux, ce qui offre l'avantage de pouvoir adresser une seconde porte logique en sortie d'un état zéro.

\section{Conclusion.}

Dans ce panorama très divers des modulateurs spatiaux de lumière actuellement commercialisés ou à l'étude, des directions d'évolution semblent manifestes.

La principale est l'utilisation croissante de la microélectronique, qui envahit même le domaine des modulateurs à adressage optique.

Un autre élément marquant est l'utilisation de modulateurs unidimensionnels, même à ondes guidées, dans des structures à connectique tridimensionnelle, où ils sont complémentaires des modulateurs à deux dimensions chaque fois qu'un calcul correspond à un changement d'espace (Vecteur vers Matrice et réciproquement).

Enfin, il apparaît que l'effort de développement industriel n'est plus motivé exclusivement par des utilisations étrangères au calcul optique parallèle. $\mathrm{Au}$ contraire, on voit apparaître un nombre croissant de dispositifs spécialement étudiés pour cet usage, spécialement les modulateurs non linéaires (à seuil ou bistables) à adressage optique.

L'ensemble de l'évolution va dans le sens d'une harmonisation des techniques employées pour tous les composants optiques actifs : émetteurs, modulateurs linéaires, composants logiques et détecteurs. Il apparaît aujourd'hui, grâce à l'emploi des semiconducteurs, une convergence vers des «technologies compatibles » non seulement entre elles, mais avec l'électronique analogique et numérique. Les recherches actuelles sur une connectique optique programmable entre VLSI ne pourront que renforcer cette tendance au rapprochement, illustrée par les expérimentations récentes de modulateurs spatiaux « tout$\mathrm{Si}$ » et « tout-AsGa » qui rendent désormais inadéquate l'expression de composants logiques «toutoptiques ». 


\section{Bibliographie}

[1] Fisher, A., A review of spatial light modulators, Technical Digest of the OSA Topic. Meet. on Optical Computing, Incline Village, Nevada, March 18-20, 1985, Tu C1, 13 pages.

[2] Fisher, A., Spatial Light Modulators, à paraître dans Advances in Electronics and Electron physics.

[3] Psaltis, D., Two-dimensional optical processing using one dimensional input devices, Proc. of the IEEE 72/7 (1984) p. 962-974.

[4] Athale, R., LeE, J., Optical processing using outerproduct concepts, Proc. of the IEEE 72/7 (1984) p. 931-941.

[5] Vanderlugt, A., Moore, G., Mathe, S., Multichannel bragg cells : compensation for acoustic spreading, Appl. Opt. 22 (1983) 3906-3912.

[6] Psaltis, D., Yu, J., Hong, J., Bias-free time integrating optical correlator using a photorefractive crystal, Appl. Opt. 24 (1985) 3860-3865.

[7] VERBER, C., Integrated-optical approaches to numerical optical processing, Proc. of the IEEE 72/7 (1984) p. 942-953.

[8] JoHNSON, R. et al., Characteristics of the linear array total internal reflection (TIR) electrooptic spatial light modulator for optical information processing, Opt. Eng. 22 (1983) 665-674.

[9] KIngSTON, R., Signal correlation using a 1-D electroabsorptive CCD spatial light modulator, Proc. of the IEEE 72/7 (1984) p. 954-961.

[10] Fisher, A. et al., Optical guided-wave interactions with magnetostatic waves at microwave frequencies, Appl. Phys. Lett. 41 (1982) 779-781.

[11] Soffer, B., Programmable real-time incoherent matrix multiplier for optical processing, Appl. Opt. 25 (1986) 2295-2305.

[12] Noble, M., Compact coherent light valve processor, SPIE Proc. 202 (1979) p. 137-146.

[13] SCHneEberger, B. et al., Real time spatial light modulator, Opt. Commun. 31 (1979) 13-15.

[14] Marie, G., Donjon, J., Hazan, J. P., Pockels effect devices and their applications, dans Advances in image pickup and display, Vol. 1 (Edit. B. Kazan), 1974, p. 226-302.

[15] Nasibov, A., ShemchuK, E., Use of laser electronbeam tubes in projection television, Sov. J. Quantum Electron. 8 (1978) 1082-1085.

[16] Potaturkin, O. et al., Holographic intensity correlator using a laser cathode-ray tube, Opt. Commun. 40 (1982) 164-168.

[17] Schwartz, A., Wang, X., Warde, C., Electronbeam addressed microchannel spatial light modulator, Opt. Eng. 24 (1985) 119-123.

[18] Ling, L. C., Fisher, A. et al., Operation of the photoemitter membrane spatial light modulator, SPIE Proc. 567, paper 23.

[19] Ross, W., Psaltis, D., Anderson, R., Two-dimensional magneto-optic spatial light modulator for signal processing, Opt. Eng. 22 (1983) 485-490.

[20] Pape, D., Hornbeck, L., Characteristics of the deformable mirror device for optical information processing, Opt. Eng. 22 (1983) 675-681.
[21] GREGORY, D., Real-time pattern recognition using a modified liquid crystal television in a coherent optical correlator, Appl. Opt. 25 (1986) 467-469.

[22] TAI, A., Low-cost LCD spatial light modulator with high optical quality, Appl. Opt. 25 (1986) 13801382.

[23] EFron, U. et al., Silicon liquid crystal light valves : status and issues, Opt. Eng. 22 (1983) 682-686.

[24] GoodHUE, W. et al., Quantum-well CCDs for CCD addressed multiple-quantum-well spatial light modulators, J. Vac. Sci. Technol. B 4 (1986) 769772.

[25] Johnson, R., GuÉrin, J.-M., SwANBerg, M., Scophony spatial light modulator, Opt. Eng. 24 (1985) 93-100

[26] KAHN, F., IR-laser-addressed thermo-optic smectic liquid-crystal storage displays, Appl. Phys. Lett. 22 (1973) 111-113.

[27] Hareng, M., Le Berre, S., Formation of synthetic images on a laser-beam-addressed smectic liquid-crystal display, Electron. Lett. 11 (1975).

[28] Sprague, R., Acoustooptic snapshot PROM : a real-time optical-signal spectrum analyser, Appl. Opt. 17 (1978) 2762-2767.

[29] Lebreton, G., BAMLER, R., GLUNDER, H., Imaging on thermoplastic films : a new recording technique for a real-time coherent light valve, Appl. Opt. 24 (1985) 450-452.

[30] Colburn, W., Chang, B., Photoconductor-thermoplastic image transducer, Opt. Eng. 17 (1978) 334-343.

[31] Donjon, J. et al., A Pockels-effect light valve : Phototitus. Applications to optical image processing, IEEE Trans. on Electron Dev. 20 (1973) 1037-1042.

[32] KERMISCH, D., Image formation mechanism in the gamma-Ruticon, Appl. Opt. 15 (1976) 17751786.

[33] CAIMI, F., The photodichroic alkali-halides as optical processing elements, Opt. Eng. 17 (1978) 327333.

[34] LAND, C., Optical information storage and spatial light modulation in PLZT ceramics, Opt. Eng. 17 (1978), 317-326.

[35] Aiken, G., Balaberda, R., Evaluation of PROM characteristics, Appl. Opt. 23 (1984), 901-904.

[36] Minemoto, T., Numata, S., Miyamoto, K., Optical parallel logic gate using spatial light modulators with the Pockels effect: implementation using three PROM devices, Appl. Opt. 25 (1986) 948-955.

[37] Casasent, D., Caimi, F., Khomenko, A., Test and evaluation of the Soviet PROM and PRIZ spatial light modulators, Appl. Opt. 20 (1981) 4215-4220.

[38] BleHA, W. et al., Application of the liquid crystal light valve to real-time optical data processing, Opt. Eng. 17 (1978) 371-384.

[39] Partanov, A., Kompanets, I., Popov, Y., Spatial modulation of light in photosensitive high-resolution metal-insulator-semiconductor structures 
with liquid crystals, Sov. J. Quantum Electron 10 (1980) 167-171.

[40] Aubourg, P. et al., Liquid crystal light valve using bulk monocrystalline BSO as the photoconductive material, Appl. Opt. 21 (1982) 3706-3712.

[41] Huignard, J.-P., Micheron, F., High sensitivity read-write volume holographic storage in BSO and BGO crystals, Appl. Phys. Lett. 29 (1976) 591-593.

[42] HuignaRd, J.-P. et al., Wave mixing in photorefractive BSO crystals and its applications, Opt. Eng. 24 (1985) 586-592.

[43] Fainman, Y., Klanknik, E., Lee, S., Optimal coherent image amplification by two-wave coupling in photorefractive $\mathrm{BaTiO}_{3}$, Opt. Eng. 25 (1986) 228-234.

[44] Fainman, Y., Guest, C., Lee, S., Optical digital logic operations by two-beam coupling in photorefractive material, Appl. Opt. 25 (1986) 15981603.

[45] Marrakchi, A., Tanguay, A., Yu, J., Psaltis, D., Physical characterization of the photorefractive incoherent-to-coherent optical converter, Opt. Eng. 24 (1985) 124-131.

[46] Hess, K., Dandliker, R., Modulateur spatial de lumière à surface déformable, Actes du Coll. Horizons de l'Optique 85, Besançon, p. 236.

[47] Soffer, B., Strand, T., Sawchuk, A., Chavel, P., Variable grating mode liquid crystal device for optical processing and computing, Mol. Cryst. Liq. Cryst. 70 (1981) 145-161.

[48] TANguay, A., SofFer, B. et al., Physical characterization of the variable grating mode liquid crystal device, Opt. Eng. 22 (1983) 687-694.

[49] Warde, C., Thackara, J., Operating mode of the microchannel spatial light modulator, Opt. Eng. 22 (1983) 695-703.

[50] Hara, T., Sugiyama, M., Suzuki, Y., A spatial light modulator, Proc. of the 8th Sympos. on Photoelectron Imaging devices, London, 57 Sept. 1983.

[51] HARA, T. et al., Microchannel spatial light modulator having the functions of image zooming, shifting and rotating, Appl. Opt. 25 (1986) 2306-2310.
[52] Fisher, A. et al., Photoemitter membrane light modulator, Opt. Eng. 25 (1986) 261-268.

[53] PAPE, D., Optically addressed membrane spatial light modulator, Opt. Eng. 24 (1985) 107-110.

[54] BrooKs, R., Micromechanical light modulators on silicon, Opt. Eng. 24 (1985) 101-106.

[55] LEE, S. et al., Two-dimensional silicon/PLZT spatial light modulator : design considerations and technology, Opt. Eng. 25 (1986) 250-260.

[56]Athale, R., LEe, S. et al., Bistability and thresholding by a new photoconductor-twisted nematic crystal device with optical feedback, Appl. Opt. 20 (1981) 1424-1432.

[57] Ledoux, I. et al., Highly efficient single-crystalline organic thin films for quadratic nonlinear optics, Opt. Eng. 25 (1986) 202-210.

[58] SMITH, S. et al., Nonlinear optical circuit elements as logic gates for optical computers : the first optical digital circuits, Opt. Eng. 24 (1985) 569-574.

[59] JEWELL, J. et al., 3-pJ, $82 \mathrm{MHz}$ optical logic gates in a room-temperature GaAs/AlGaAs multiplequantum-well étalon, Appl. Phys. Lett. 46 (1985) 918-920.

[60] Ovadia, S. et al., Evidence that room temperature optical bistability is excitonic in both bulk and multiple-quantum-well gallium arsenide, Opt. Eng. 24 (1985) 565-568.

[61] Chemla, D., Miller, D., SMith, P., Nonlinear optical properties of $\mathrm{GaAs} / \mathrm{GaAlAs}$ multiple quantum well material : phenomena and applications, Opt. Eng. 24 (1985) 556-564.

[62] OJimA, M. et al., Optical Nor gate using diode laser sources, Appl. Opt. 25 (1986) 2311-2313.

[63] LEE, Y. H. et al., Streak-camera observation of 200ps recovery of an optical gate in a windowless GaAs etalon array, Appl. Phys. Lett. 48 (1986) 754-756.

[64] DoHLER, G., Light generation, modulation and amplification by n-i-p-i doping superlattices, $O p t$. eng. 25 (1986) 211-218.

[65] Dagenais, M., Sharfin, W., Extremely low switching energy optical bistable devices, Opt. Eng. 25 (1986) 219-224.

[66] Lohmann, A., Polarization and optical logic, Appl. Opt. 25 (1986) 1594-1597. 